\title{
Functional Polymorphisms Associated with Disease-Free Survival in Resected Carcinoma of the Esophagus
}

\author{
Jurjen J. Boonstra $\cdot$ Ronald van Marion • \\ Hugo W. Tilanus • Winand N. M. Dinjens
}

Received: 10 August 2010 / Accepted: 17 September 2010 /Published online: 5 October 2010

(C) The Author(s) 2010. This article is published with open access at Springerlink.com

\begin{abstract}
Purpose The aim of this study was to determine whether clinical outcome after surgical resection of esophageal adenocarcinoma (EAC) or esophageal squamous cell carcinoma (ESCC) could be predicted by functional polymorphisms in different proto-oncogenes and tumor suppressor genes.

Experimental Design Six single nucleotide polymorphisms (SNPs) in the AURKA (rs2273535), ERBB2 (rs1136201), MDM2 (rs2279744), CDH1 (rs5030625), CDKN2A (rs11515), and TP73 (rs2273953) genes were genotyped in a consecutive cohort of 346 esophageal cancer patients, who had underwent surgical resection with curative intent. Associations with disease-free survival (DFS) were analyzed with Kaplan-Meier curves and Cox regression, adjusting for potential confounders.

Results Univariate analysis showed no significant associations between the tested polymorphisms and DFS in patients with EAC or ESCC. However, in a multivariate analysis, patients with EAC carrying the heterozygous MDM2 (rs2279744) T/G genotype had significantly improved DFS compared with patients carrying the wild-type genotype (adjusted hazard ratio (AHR), 0.63; 95\% confidence interval (CI) [0.45-0.88]). Patients with EAC harboring the homozygous CDH1 (rs5030625) GA/GA genotype had a significantly reduced survival as compared with patients carrying the wild-type genotype AHR 4.0, 95\% CI [1.4-11].

Conclusions In a large cohort of esophageal cancer patients, the MDM2 T/G and CDH1 GA/GA genotype confer risk of death in patients with EAC. These data suggest that inter-individual differences in germ-line DNA have an impact on DFS in patients with EAC.
\end{abstract}

Keywords Esophagus $\cdot$ Adenocarcinoma $\cdot$ Squamous cell carcinoma $\cdot$ Polymorphism $\cdot$ SNP

Financial Support This study was supported by the revolving fund (FC0991) of the Erasmus MC Rotterdam and from the SK foundation.

J. J. Boonstra $(\bowtie) \cdot$ H. W. Tilanus

Department of Surgery, Erasmus MC,

University Medical Center Rotterdam,

P.O. Box 2040, 3000 CA Rotterdam, The Netherlands

e-mail: j.j.boonstra@erasmusmc.nl

J. J. Boonstra $\cdot$ R. van Marion - W. N. M. Dinjens

Department of Pathology, Josephine Nefkens Institute,

Room Be320a, Erasmus MC, University Medical Center

Rotterdam,

Dr. Molewaterplein 50

3015 GE Rotterdam, The Netherlands

\section{Introduction}

Many therapeutic options are used to treat esophageal cancer, but traditionally, surgery is used most frequently to obtain loco-regional control and long-term survival. ${ }^{1,2}$ Comprehensive preoperative staging has improved selection of patients for potentially curative surgery; however, many patients present with recurrent disease within 2 years after operation. The majority of these patients develop not only loco-regional recurrences, but also distant metastases (such as liver, lung, pleural, and/or peritoneal disease recurrences) are common. ${ }^{3-5}$ Despite attempts to improve the outcome of patients with esophageal cancer, prognosis remains poor with a 5 -year overall survival of $20-30 \%$. ${ }^{5,6}$

Well-known prognostic factors for esophageal cancer are summarized in tumor node metastasis (TNM) staging. ${ }^{7,8}$ Although TNM parameters have the advantage of simplic- 
ity, they do not seem to completely reflect the biologic diversity of esophageal cancer. ${ }^{9,10}$ The true drivers of this clinical biologic diversity include the molecular aberrations of the cancer and the genetic make-up of the patient. In this respect, the study of host genetic variability offers worthwhile potential to identify individuals that may have the best chance of survival. Single nucleotide polymorphisms (SNPs) in the germ-line are the most common type of host genetic variations. Gene-related functional SNPs can potentially lead to differences in protein expression and/or function. In this way, SNPs in proto-oncogenes and tumor suppressor genes can potentially alter the risk for metastatic or aggressive tumor, resulting in differences in clinical outcome.

Altered expression of the AURKA, ERBB2, MDM2, $\mathrm{CDH} 1, \mathrm{CDKN} 2 \mathrm{~A}$, and TP73 proteins has been correlated to disease progression and clinical outcome in patients with esophageal cancer. ${ }^{11-17}$ In addition, polymorphisms with effects on protein function have been identified in these proto-oncogenes and tumor suppressor genes. ${ }^{18-23}$ Based on these results, we postulated that functional SNPs in the AURKA (AURKA_NM_003600.2; rs2273535 c.449 T>A), ERBB2 (ERBB2_NM004448.2; rs1136201 c.655 A>G), MDM2 (MDM2_NM002392.2; rs2279744 $309 \mathrm{~T}>\mathrm{G})$, CDH1 (CDH1_NM004360.3; rs5030625 -347 G>GA), $C D K N 2 A\left(C D \bar{K} N 2 A \_\mathrm{NM} 000077.3\right.$; rs11515 c.712 C>G), and TP73 (TP73_NM005427.1; rs2273953 $81 \mathrm{G}>\mathrm{A}$ ) genes may serve as molecular markers for clinical outcome in patients with esophageal adenocarcinoma (EAC) or esophageal squamous cell carcinoma (ESCC) who underwent surgical resection.

\section{Patients and Methods}

Patients Between 1996 and 2001, a total of 632 consecutive patients with esophageal cancer were evaluated for surgery with curative intent at the Erasmus University Medical Center (Fig. 1). Outcome for all patients with esophageal cancer referred to our hospital are collected

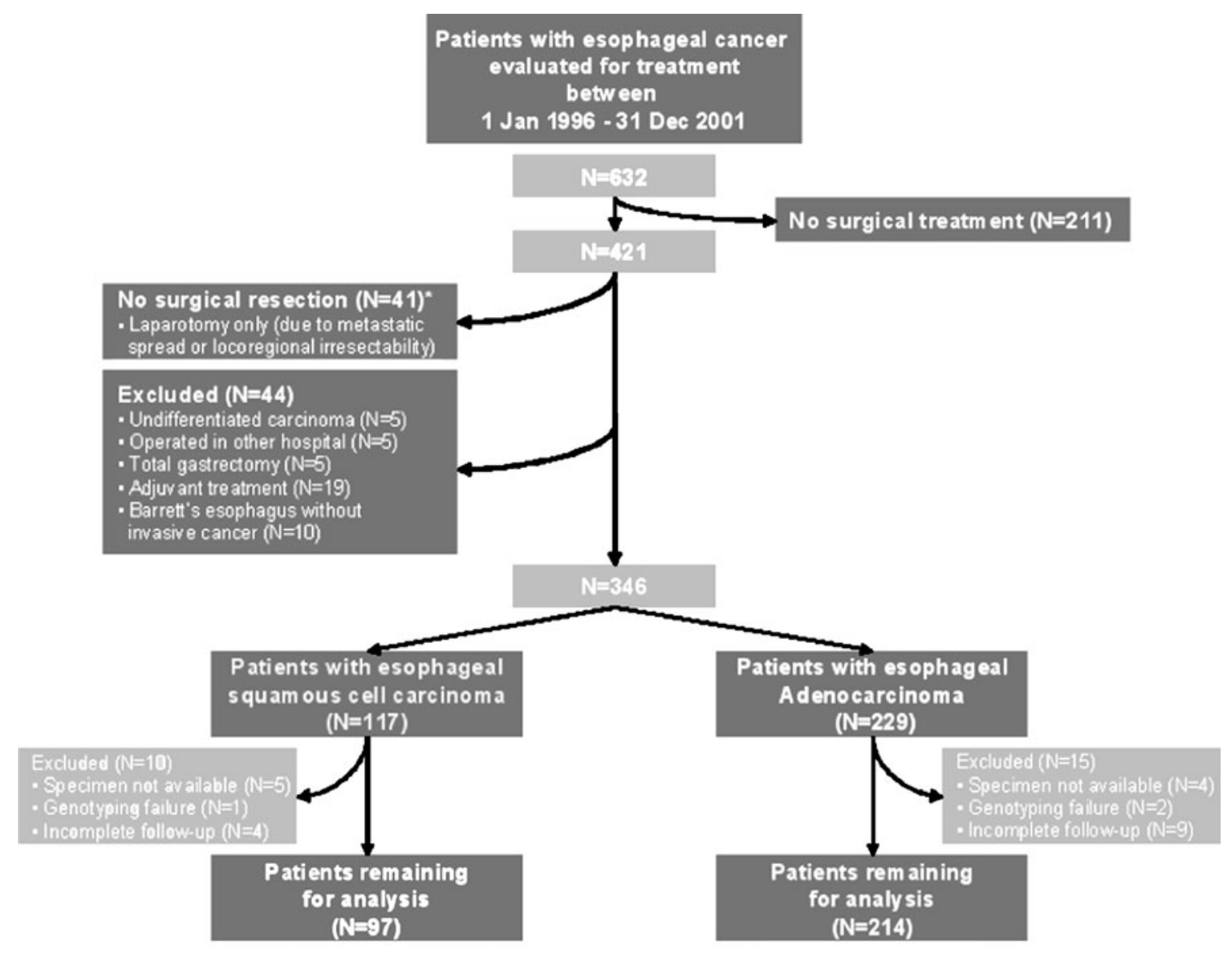

*Three patients were resected despite the presence of distant metastases

Fig. 1 Flowchart of patients with esophageal cancer referred to the Erasmus MC for treatment between January 1996 and December 2001. Patients excluded from the present study are shown 
prospectively and stored in a database by a data manager. The data collected encompassed all relevant diagnostic tests, scheduled treatments, and pathology. All patients were staged using esophago-gastroscopy with biopsies, ultrasonography of the cervical and upper abdominal region, and computed tomography of the thorax and abdomen. Endoscopic ultrasonography for evaluation of T-stage and nodal status was routinely performed.

Surgery For carcinomas of the upper half of the intrathoracic esophagus, a right-sided thoracotomy was performed. For carcinomas of the lower half of the intrathoracic esophagus, a transhiatal esophagectomy was preferred. The tumor and its adjacent lymph nodes were dissected en bloc; however, no extended lymph node dissection was performed. The continuity of the digestive tract was restored by means of a gastric tube reconstruction or colonic interposition with a cervical anastomosis. Resections were considered radical (R0) if microscopic examination revealed no tumor tissue at or less than $1 \mathrm{~mm}$ from the circumferential, proximal, or distal margins. Pathological staging was done according to the Union for International Cancer Control (UICC) sixth edition. The tumor stage after resection was classified according to the TNM classification of the International Union Against Cancer.

SNP Genotyping To determine the individual genotype for each SNP, genomic DNA was extracted from frozen or formalin-fixed and paraffin-embedded (FFPE) tissues. Normal tissue was obtained from the resection specimens (i.e., tumor-negative lymph nodes or tumor-negative resection margins). All the archival tissue samples were used according to the code for adequate secondary use of tissue, code of conduct: "Proper Secondary Use of Human Tissue" established by the Dutch Federation of Medical Scientific Societies (http://www.federa.org).

Polymerase chain reactions (PCR) were carried out in a volume of $15 \mu \mathrm{l}$ containing genomic DNA, 8.3 $\mu \mathrm{l}$ $\mathrm{H}_{2} \mathrm{O}, 5 \mu \mathrm{Mg}^{2+}$ free buffer, $25 \mathrm{mM} \mathrm{MgCl}_{2}, 0.3 \mu \mathrm{l}$ of $10 \mathrm{mM}$ deoxynucleotide triphosphates, 20 pmol of each primer, and $1 \mathrm{U}$ Taq polymerase (Promega, Madison WI, USA). PCR conditions were standardized at 35 cycles of $95^{\circ} \mathrm{C}$ for $45 \mathrm{~s}, 61^{\circ} \mathrm{C}$ for $45 \mathrm{~s}, 72^{\circ} \mathrm{C}$ for $30 \mathrm{~s}$, with a 10 -min extension at $72^{\circ} \mathrm{C}$ for $10 \mathrm{~min}$ following the last cycle. PCR primers for each SNP are shown in Table 1. For the polymorphism in CDH1 (rs5030625), amplified PCR products were visualized on a denaturing polyacrylamide gel. For detection of the restriction length polymorphisms in ERBB2 (rs1136201) and AURKA (rs2273535), PCR products were digested for $16 \mathrm{~h}$ at the appropriate temperature with $10 \mathrm{U}$ of restriction endonuclease BsmAI, MspI, or APOI (Promega, Madison, WI, USA), respec-

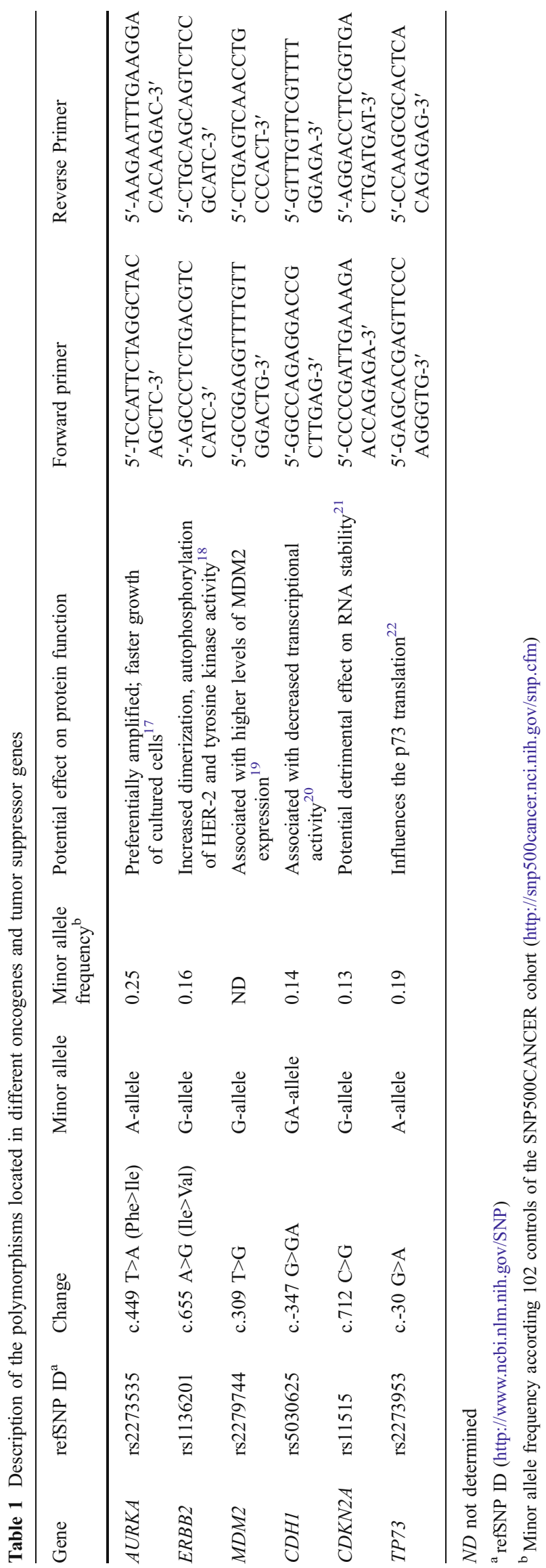


Table 2 Survival according to patients' and tumor characteristics

\begin{tabular}{|c|c|c|c|c|c|c|}
\hline \multirow[t]{2}{*}{ Variable } & \multicolumn{3}{|c|}{ Patients with EAC $(N=214)$} & \multicolumn{3}{|c|}{ Patients with ESCC $(N=97)$} \\
\hline & No. $(\%)$ & Median DFS & $P$ values & No. $(\%)$ & Median DFS & $P$ values \\
\hline Age in years & & & 0.23 & & & 0.33 \\
\hline$<65$ years & $111(52)$ & 19 & & $64(66)$ & 21 & \\
\hline$\geq 65$ years & $103(48)$ & 12 & & $33(34)$ & 12 & \\
\hline Gender & & & 0.41 & & & 0.44 \\
\hline Male & $182(85)$ & 16 & & $58(60)$ & 15 & \\
\hline Female & $32(15)$ & 11 & & $39(40)$ & 20 & \\
\hline Weight loss before operation & & & 0.013 & & & 0.47 \\
\hline No loss or $<5 \%$ & $127(59)$ & 19 & & $46(47)$ & 15 & \\
\hline $5-10 \%$ & $35(16)$ & 11 & & $31(32)$ & 27 & \\
\hline$>10 \%$ & $32(15)$ & 8 & & $17(18)$ & 10 & \\
\hline Not recorded & $20(10)$ & 10 & & $3(3)$ & 5 & \\
\hline Smoking status & & & 0.98 & & & 0.70 \\
\hline Current smoker & $54(25)$ & 14 & & $43(44)$ & 16 & \\
\hline No current smoker & $146(68)$ & 15 & & $47(49)$ & 20 & \\
\hline Not recorded & $14(7)$ & 8 & & $7(7)$ & 4 & \\
\hline Location of tumor & & & 0.150 & & & 0.008 \\
\hline Upper one third thoracic esophagus & & & & $3(3)$ & 4 & \\
\hline Middle one third thoracic esophagus & $3(1)$ & 11 & & $39(40)$ & 12 & \\
\hline Lower one third thoracic esophagus & $68(32)$ & 24 & & $45(47)$ & 20 & \\
\hline GEJ & $86(40)$ & 13 & & $10(10)$ & 60 & \\
\hline Gastric cardia & $57(27)$ & 12 & & & & \\
\hline Tumor length (cm) & & & 0.028 & & & 0.27 \\
\hline $0-2$ & $34(16)$ & 41 & & $8(8)$ & 37 & \\
\hline $3-4$ & $52(24)$ & 15 & & $24(25)$ & 10 & \\
\hline $4-5$ & $67(31)$ & 14 & & $31(32)$ & 24 & \\
\hline$\geq 6$ & $46(22)$ & 9 & & $25(26)$ & 9 & \\
\hline Not recorded & $15(7)$ & 16 & & $9(9)$ & 11 & \\
\hline Barrett's epithelium & & & 0.086 & & & \\
\hline No & $127(59)$ & 12 & & & & \\
\hline Yes & $87(41)$ & 24 & & & & \\
\hline Treatment & & & 0.83 & & & 0.18 \\
\hline Surgery alone & $180(84)$ & 14 & & $28(29)$ & 8 & \\
\hline Chemotherapy+surgery & $23(11)$ & 15 & & $65(67)$ & 15 & \\
\hline Chemoradiotherapy + surgery & $11(5)$ & 21 & & $4(4)$ & Not reached & \\
\hline Resection type & & & 0.83 & & & 0.042 \\
\hline Transhiatal & $187(87)$ & 15 & & $53(55)$ & 37 & \\
\hline Transthoracic & $27(13)$ & 9 & & $44(45)$ & 11 & \\
\hline Post-operative UICC stage & & & $<0.001$ & & & $<0.001$ \\
\hline Complete response & $8(4)$ & 40 & & $13(13)$ & Not reached & \\
\hline I & $24(11)$ & 98 & & $13(13)$ & 86 & \\
\hline IIA & $43(20)$ & 37 & & $34(35)$ & 12 & \\
\hline IIB & $8(4)$ & 15 & & $4(4)$ & 26 & \\
\hline III & $74(34)$ & 11 & & $17(18)$ & 8 & \\
\hline IV & $57(27)$ & 7 & & $16(17)$ & 4 & \\
\hline Radicality of resection & & & $<0.001$ & & & $<0.001$ \\
\hline R0 & $141(66)$ & 34 & & $65(67)$ & 41 & \\
\hline $\mathrm{R} 1$ & $70(33)$ & 7 & & $29(30)$ & 6 & \\
\hline R2 & $3(1)$ & 9 & & $3(3)$ & 5 & \\
\hline
\end{tabular}


tively. The DNA fragments were separated using 3\% agarose gels. The polymorphisms in CDKN2A (rs11515), MDM2 (rs2279744), and TP73 (rs2273953) were genotyped by bi-directional sequencing.

Statistical Analysis Data on follow-up were collected from the prospective database and the medical charts. All patients were followed at an interval of 3 to 4 months during the first year, every 6 months for the second year, and then at the end of each year until 5 years after treatment. Recurrence or disease progression was diagnosed on clinical grounds. Whenever a relapse was suspected, radiologic, endoscopic, or histologic confirmation was sought. Recurrent disease was classified as local-regional (occurring in the upper abdomen or mediastinum) or distant (including cervical recurrences).

Study end-point was disease-free survival (DFS) that was defined as the time from surgery until recurrent disease or death from any cause. The Kaplan-Meier survival function and log-rank tests were used to assess clinical outcome in relation to patient's characteristics and individual polymorphisms. Cox proportional hazard ratios for patients with EAC were adjusted for weight loss prior to operation, tumor length, presence of Barrett's epithelium, radicality of resection, and pathological tumor stage. For patients with ESCC, Cox proportional hazard ratios were adjusted for location of tumor, resection type, post-operative TNM stage, and radicality of resection. Statistical significance was set at the $5 \%$ level. We did not adjust for multiple testing since each gene outcome was prespecified and of interest in itself.

\section{Results}

Patients A total of 346 esophageal cancer patients underwent surgical resection with curative intent. Of these, 25

Table 3 Polymorphisms and clinical outcome in patients with resected EAC

\begin{tabular}{|c|c|c|c|c|c|c|c|c|c|c|}
\hline \multirow[t]{2}{*}{ Genotype } & \multicolumn{5}{|c|}{ Disease-free survival in EAC patients } & \multicolumn{5}{|c|}{ Disease-free survival in ESCC patients } \\
\hline & $\mathrm{N}$ & MPS (months) & Log-rank $P$ & AHR & {$[95 \% \mathrm{CI}]^{\mathrm{a}}$} & $\mathrm{N}$ & MPS (months) & Log-rank $P$ & AHR & {$[95 \% \mathrm{CI}]^{\mathrm{b}}$} \\
\hline \multicolumn{11}{|c|}{$A U R K A \_r s 2273535$} \\
\hline $\mathrm{T} / \mathrm{T}$ & 129 & 15 & 0.83 & \multicolumn{2}{|c|}{ Reference } & 62 & 12 & 0.72 & \multicolumn{2}{|c|}{ Reference } \\
\hline $\mathrm{A} / \mathrm{T}$ & 75 & 14 & & 1.1 & {$[0.76-1.4]$} & 29 & 20 & & 0.60 & {$[0.17-2.1]$} \\
\hline $\mathrm{A} / \mathrm{A}$ & 9 & 21 & & 0.92 & {$[0.42-2.0]$} & 5 & 55 & & 0.63 & {$[0.18-2.1]$} \\
\hline \multicolumn{11}{|c|}{$E R B B 2$ 2_s 1136201} \\
\hline $\mathrm{A} / \mathrm{A}$ & 113 & 14 & 0.25 & \multicolumn{2}{|c|}{ Reference } & 66 & 12 & 0.66 & \multicolumn{2}{|c|}{ Reference } \\
\hline $\mathrm{A} / \mathrm{G}$ & 86 & 15 & & 0.92 & {$[0.67-1.3]$} & 23 & 26 & & 0.73 & [0.41-1.3] \\
\hline $\mathrm{G} / \mathrm{G}$ & 14 & 12 & & 0.68 & {$[0.33-1.4]$} & 6 & 8 & & 1.3 & [0.49-3.2] \\
\hline \multicolumn{11}{|c|}{$M D M 2 \_r s 2279744$} \\
\hline $\mathrm{T} / \mathrm{T}$ & 100 & 11 & 0.076 & \multicolumn{2}{|c|}{ Reference } & 40 & 10 & 0.63 & \multicolumn{2}{|c|}{ Reference } \\
\hline $\mathrm{T} / \mathrm{G}$ & 84 & 19 & & 0.63 & {$[0.45-0.88]$} & 45 & 21 & & 0.98 & {$[0.59-1.6]$} \\
\hline $\mathrm{G} / \mathrm{G}$ & 24 & 12 & & 0.95 & {$[0.58-1.6]$} & 7 & 16 & & 0.81 & [0.28-2.4] \\
\hline \multicolumn{11}{|c|}{ CDH1_rs5030625 } \\
\hline $\mathrm{G} / \mathrm{G}$ & 166 & 17 & 0.14 & & & 68 & 11 & 0.13 & \multicolumn{2}{|c|}{ Reference } \\
\hline G/GA & 41 & 11 & & 1.2 & {$[0.78-1.7]$} & 18 & 27 & & 0.63 & [0.32-1.3] \\
\hline GA/GA & 4 & 7 & & 4.0 & {$[1.4-11]$} & 1 & Not reached & & & \\
\hline \multicolumn{11}{|c|}{$C D K N 2 A \_r s 11515$} \\
\hline $\mathrm{C} / \mathrm{C}$ & 162 & 13 & 0.79 & \multicolumn{2}{|c|}{ Reference } & 74 & 12 & 0.67 & \multicolumn{2}{|c|}{ Reference } \\
\hline $\mathrm{C} / \mathrm{G}$ & 47 & 19 & & 0.94 & {$[0.65-1.3]$} & 20 & 20 & & 0.67 & [0.36-1.3] \\
\hline $\mathrm{G} / \mathrm{G}$ & 4 & 19 & & 1.7 & {$[0.52-5.6]$} & 1 & Not reached & & & \\
\hline \multicolumn{11}{|c|}{ TP73_rs2273953 } \\
\hline $\mathrm{G} / \mathrm{G}$ & 138 & 16 & 0.44 & \multicolumn{2}{|c|}{ Reference } & 62 & 24 & 0.48 & \multicolumn{2}{|c|}{ Reference } \\
\hline $\mathrm{G} / \mathrm{A}$ & 69 & 13 & & 0.98 & {$[0.71-1.4]$} & 32 & 10 & & 1.1 & [0.66-1.8] \\
\hline $\mathrm{A} / \mathrm{A}$ & 5 & 11 & & 1.1 & {$[0.41-3.1]$} & 2 & 4 & & 1.7 & [0.40-7.3] \\
\hline
\end{tabular}


A

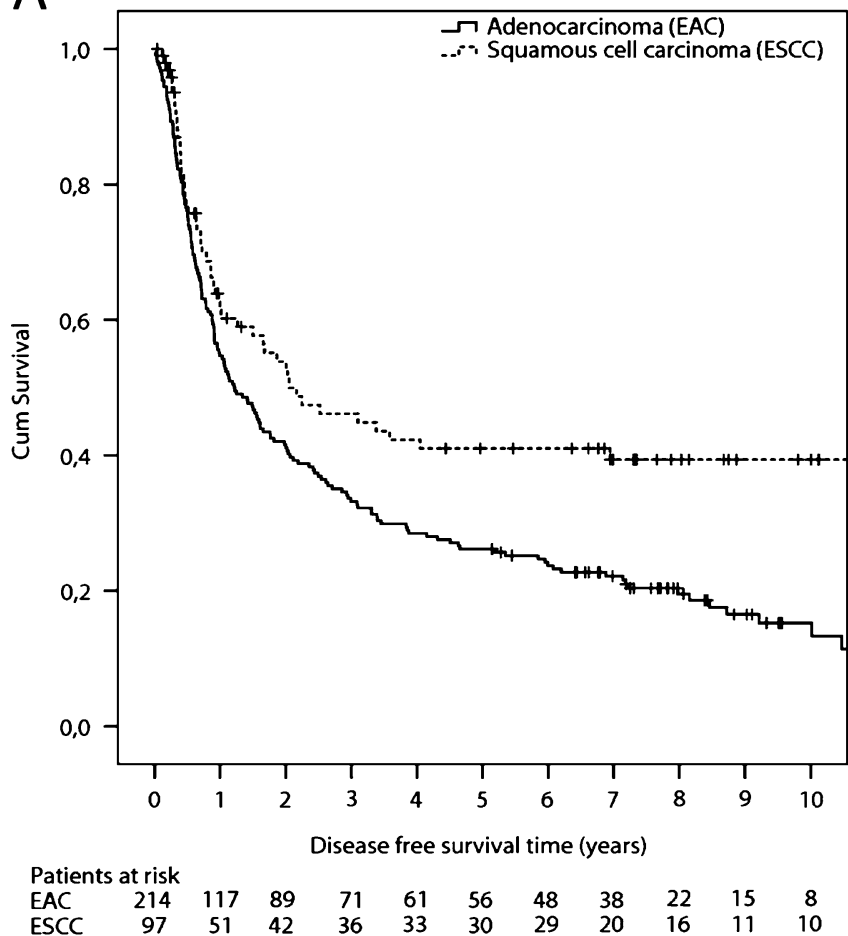

B

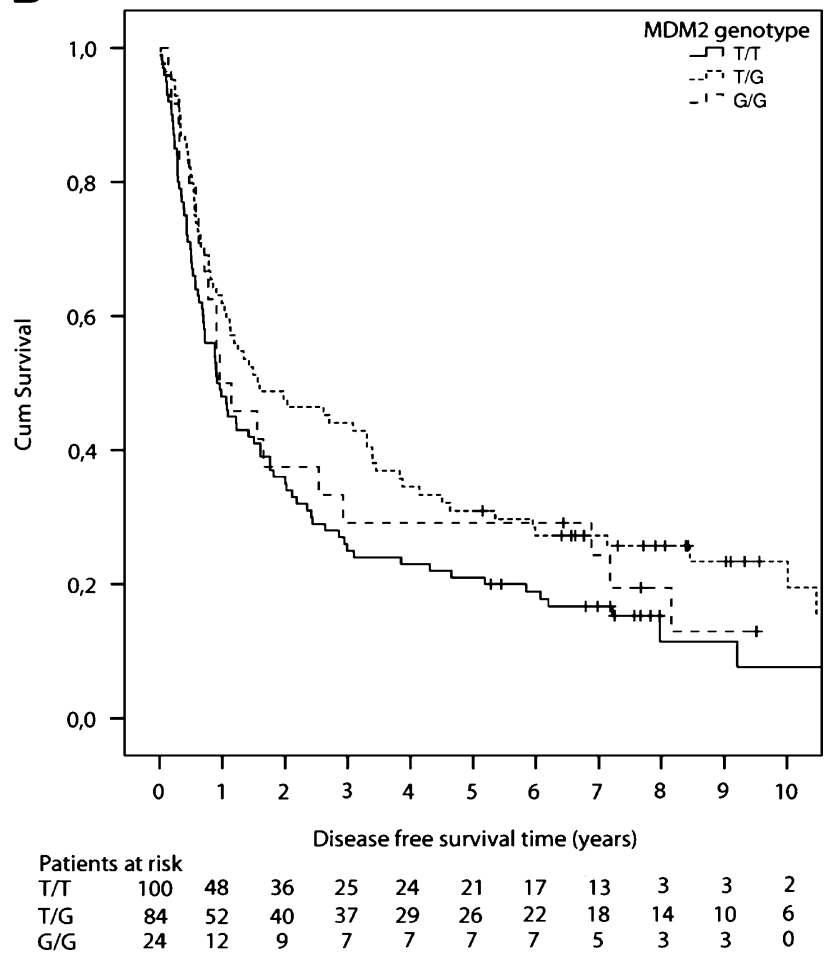

C

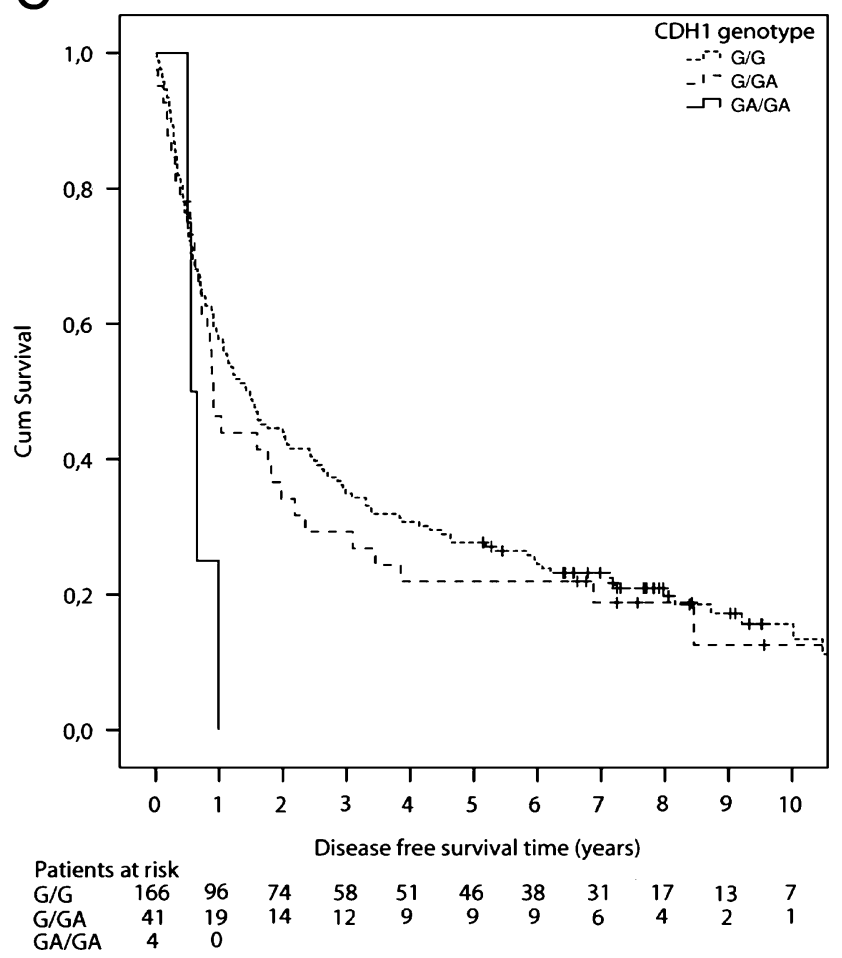

Fig. 2 a Kaplan-Meier analysis of DFS in patients with esophageal cancer, by histological subgroup. b Kaplan-Meier analysis of DFS in patients with EAC, by MDM2 (rs2279744). c Kaplan-Meier analysis of DFS in patients with EAC, by CDH1 genotype (rs5030625)

patients were excluded from the current follow-up study because no tissue samples were available $(N=9)$, genotyping failure $(N=3)$, or incomplete follow-up $(N=13)$ (Fig. 1). Of the $214 \mathrm{EAC}$ and $97 \mathrm{ESCC}$ patients remaining for analysis, the majority were male, $85 \%$ in EAC and $60 \%$ in ESCC. Median age at time of diagnosis was 64 and 61 years, respectively. Of all patients with EAC, 84\% underwent primary surgery and $16 \%$ received preoperative 
chemotherapy with or without radiotherapy. In contrast, $71 \%$ of patients with ESCC received preoperative chemotherapy with or without radiotherapy and $29 \%$ underwent primary surgical resection (Table 2).

SNP Genotyping Genotyping was complete in $95 \%$ to $100 \%$ of EAC and ESCC patients. The genotype distributions did not deviate from HWE $(P>0.05)$. The genotype distribution of each SNP is listed in Table 3. Tumor stage distributions were similar across all SNP genotypes, and there was no association between genotypes and age at diagnosis, sex, weight loss, smoking status, or preoperative treatment.

DFS and Pattern of Disease Recurrence The median DFS of EAC patients was 14 months (range, 0.07-138 months) and for ESCC patients 16 months (range, 0.5-148 months). At the time of analysis, 37 (17\%) EAC and 27 (28\%) ESCC patients were alive with a median DFS time of 93 months (range, 62-138 months) and 104 months (range 79148 months), respectively (Fig 2A).

The pattern of disease recurrence is depicted in Table 3. Loco-regional recurrences were mediastinal or abdominal lymph node metastases and recurrences in the gastric tube. Distant metastases were found in liver, lung, brain, bone, adrenal gland, pleura, peritoneum, and skin.

Recurrent disease after surgery was found in $138(78 \%)$ EAC patients; 40 patients had loco-regional recurrence, 51 had distant metastasis, and 46 had both loco-regional recurrence and distant metastasis. One patient had disease recurrence, but the site of failure was not recorded. Diseases recurrences were found in 51 ESCC patients; 28 patients had loco-regional recurrence, 10 had distant metastasis, and 10 had both loco-regional recurrence and distant metastasis. Three patients had disease recurrence, but site of failure was not recorded.

SNP Genotype and DFS Univariate analysis showed no significant associations between DFS in patients with EAC or ESCC and the genotype distributions of the AURKA, ERBB2, MDM2, CDH1, CDKN2A, and the TP73 gene polymorphisms (Table 4; Fig $2 \mathrm{~B}$ and $\mathrm{C}$ ). However, in a multivariate analysis, patients with EAC carrying the heterozygous MDM2 (rs2279744) T/G genotype had significantly improved DFS compared with patients carrying the wild-type $\mathrm{T} / \mathrm{T}$ genotype (adjusted hazard ratio (AHR) $0.63,95 \%$ confidence interval (CI) [0.45-0.88], $P=$ 0.007). The post-operative TNM stage of the tumor and the radicality of resection were also found important factors for DFS (HR 1.4, 95\% CI [1.2-1.6], $P<0.0001$ and HR 2.3, 95\%CI [1.7-3.1], $P<0.0001$ respectively).

Also, patients with EAC harboring the homozygous CDH1 (rs5030625) GA/GA genotype had a significantly
Table 4 Pattern of failure

\begin{tabular}{lcc}
\hline & EAC $(N=214)$ & ESCC $(N=97)$ \\
\hline Alive & $37(17)$ & $27(28)$ \\
$\begin{array}{l}\text { Nature of first failure } \\
\text { Local recurrence }\end{array}$ & $40(29)$ & $28(55)$ \\
$\begin{array}{l}\text { Distant metastases } \\
\text { Local recurrence and } \\
\text { distant metastases }\end{array}$ & $51(37)$ & $10(20)$ \\
$\begin{array}{l}\text { Disease recurrence but } \\
\text { site of failure not reported }\end{array}$ & $46(33)$ & $10(20)$ \\
$\begin{array}{l}\text { Total deaths } \\
\text { Cause of death }\end{array}$ & $17(1)$ & $3(5)$ \\
Cancer-related & $138(78)$ & $70(72)$ \\
$\begin{array}{l}\text { Surgery-related } \\
\text { 2nd Primary }\end{array}$ & $14(8)$ & $51(71)$ \\
Death from other cause & $5(3)$ & $7(11)$ \\
$\quad$ not cancer-related) & $20(11)$ & $6(8)$ \\
\hline
\end{tabular}

reduced survival as compared with patients carrying the wild-type G/G genotype AHR 4.0, 95\% CI [1.4-11], $P=$ 0.008 . In multivariate analysis, the post-operative TNM stage of the tumor and the radicality of resection were found as important factors for DFS (HR 1.4, 95\% CI [1.21.5], $P<0.0001$ and HR 2.4, 95\%CI [1.7-3.2], $P<0.0001$ respectively).

\section{Discussion}

In the present study, we determined the relationship between inter-individual DNA variations in six bona fide proto-oncogenes and tumor suppressor genes and DFS in a large cohort of Caucasian patients with esophageal cancer. After adjustment for potential confounders, the variant genotypes of SNPs located in the promoter region of the $M D M 2$ and $C D H 1$ gene were significantly associated with DFS in patients with EAC.

The results of the present study showed a significant survival benefit for patients harboring the MDM2 T/G as compared with patients carrying the wild-type T/T genotype. The MDM2 protein is a nuclear phosphoprotein that binds and inhibits the tumor suppressor TP53 as part of an autoregulatory negative feedback loop. The most intensively characterized MDM2 polymorphism is the $\mathrm{T} 309 \mathrm{G}$ promoter SNP located in the first intron. ${ }^{20}$ The $\mathrm{G}$ variant of this SNP is known to increase promoter-binding affinity, leading to upregulation of MDM2 and consequent inhibition and downregulation of the p53 pathway. Therefore, it could be expected that the variant MDM2 genotypes $(\mathrm{T} / \mathrm{G}$ and $\mathrm{G} / \mathrm{G})$ 
are associated with adverse outcome in esophageal cancer patients (as shown in other cancer types). ${ }^{24}$ However, the present study showed improved survival in patients with the MDM2 T/G genotype compared with the wild-type T/T genotype. A possible explanation for our findings is provided by a large study in breast cancer patients that reported strong interaction between the MDM2 SNP status and tumor TP53 status, which appeared to modify the association between TP53 status and breast cancer survival. ${ }^{25}$ Among breast cancer patients with the wild-type MDM2 genotype (T/T), a mutant TP53 status and aberrant TP53 expression in breast tumors were associated with poor survival. The tumor TP53 status was not associated with breast cancer survival among carriers of the variant MDM2 allele (T/G or $\mathrm{G} / \mathrm{G})$. Since TP53 is the most frequently mutated gene in EAC, it could be hypothesized that the tumors of most patients with the T/T genotype harbor a TP53 mutation, which could lead to a reduced survival as observed in the present study. In a previously well-conducted study, the known TP53 codon 72 Arg/Pro and $M D M 2$ polymorphisms were genotyped in 300 patients with EAC and 63 patients with ESCC. ${ }^{26}$ As in concordance with the results of the present study, patients with EAC harboring the MDM2 T/G genotype had a borderline improved overall survival as compared with patients carrying the wild-type genotype (AHR for death $0.70,95 \%$ CI [0.50-0.99], $P=0.04$ ). But unlike the present study, the MDM2 variant genotype did correlate with marked reduced survival in patients with ESCC. This could be due to differences in study samples size, population selection, tissue handling, and genotyping methods.

In this study, patients carrying the CDH1 GA/GA genotype had a significantly reduced survival as compared with patients with the wild-type G/G genotype. However, it should be noted that only four EAC patients carried the GA/GA genotype, which may represents a chance finding. Nevertheless, this $-347 \mathrm{G} / \mathrm{GA}$ insertion polymorphism located in the promoter of the cell-cell adhesion gene $C D H 1$ has been reported to suppress $C D H 1$ gene expression and was found to be associated with familial gastric cancer and sporadic colorectal cancer. ${ }^{27}$ The GA-allele has been associated with significant suppression of $\mathrm{CDH} 1$ promoter activity in colorectal and gastric cancer cell lines. ${ }^{27}$ It can be hypothesized that the GA-allele might enhance the progression of esophageal cancer by reducing $\mathrm{CDH} 1$ transcription resulting in a decrease in $\mathrm{CDH} 1$ protein expression and impairment of cell-cell adhesion. All four patients harboring the GA/GA genotype died of recurrent disease; three had loco-regional and distant metastasis, and one had only locoregional disease recurrence.

To our knowledge, this is one of the first studies that investigated the relationship between polymorphisms and esophageal cancer outcome. Here, we studied (only) six polymorphisms, whereas SNP arrays can determine more than a million of SNPs in one sample. Although this technique is widely used on blood or fresh frozen samples, it is not very suitable for FFPE tissue samples (our series consisted primarily of FFPE samples). Therefore, collection of blood samples or fresh frozen tissue samples of esophageal cancer patients is necessary and should become standard procedure in order to perform genome-wide association studies. In this study, the majority of polymorphisms were not associated with DFS after esophagectomy. It could be well that our study, among a relatively large population $(N=346)$ of esophageal cancer patients, failed to observe a difference due to under powering. Since esophageal cancer has a relatively low incidence, consortia (of multiple hospitals) are needed to validate these associations.

Recurrent cancer is the leading cause of death in patients undergoing surgical resection. ${ }^{3-5}$ Although treatment options for esophageal cancer recurrences are limited, it could be proposed that early detection of recurrent disease is desirable because aggressive treatment may result in prolonged tumor-free survival or occasional cure. In this light, our findings could contribute to the identification of patients who are at high or low risk for the development of disease recurrences. It can also be proposed that patients with a certain genetic constitution that is associated with a high chance of (distant) disease recurrence should be given systemic adjuvant treatment after surgical resection. Furthermore, identification of polymorphisms associated with DFS could serve well as hypothesis generating for prospective studies that evaluate the prognostic significance of germ-line variants.

In summary, our results indicate that two of six investigated functional polymorphisms are associated with DFS in patients who underwent esophagectomy for EAC. Patients with EAC carrying the heterozygous MDM2 T/G genotype had twofold reduced risk of disease recurrence, and patients with the homozygous $\mathrm{CDH} 1 \mathrm{GA} / \mathrm{GA}$ had a fourfold increased risk of disease recurrence. Additional prospective studies are necessary to validate both associations and to study the prognostic significance of both germline variants.

Acknowledgements We thank C. Vollebregt, L. Koppert and H. van Dekken for the assistance in collecting clinical and pathological data.

Conflicts of Interest There are no conflicts of interest.

Open Access This article is distributed under the terms of the Creative Commons Attribution Noncommercial License which permits any noncommercial use, distribution, and reproduction in any medium, provided the original author(s) and source are credited. 


\section{References}

1. Mariette C, Piessen G, Triboulet JP. Therapeutic strategies in oesophageal carcinoma: role of surgery and other modalities. Lancet Oncol 2007; 8:545-53.

2. $\mathrm{Wu} \mathrm{PC}$, Posner MC. The role of surgery in the management of oesophageal cancer. Lancet Oncol 2003; 4:481-8.

3. Hulscher JB, van Sandick JW, Tijssen JG, Obertop H, van Lanschot JJ. The recurrence pattern of esophageal carcinoma after transhiatal resection. J Am Coll Surg 2000; 191:143-8.

4. Mariette C, Balon JM, Piessen G, Fabre S, Van Seuningen I, Triboulet JP. Pattern of recurrence following complete resection of esophageal carcinoma and factors predictive of recurrent disease. Cancer 2003; 97:1616-23.

5. Kelsen DP, Winter KA, Gunderson LL, et al. Long-term results of RTOG trial 8911 (USA Intergroup 113): a random assignment trial comparison of chemotherapy followed by surgery compared with surgery alone for esophageal cancer. J Clin Oncol 2007; 25:3719-25.

6. Medical Research Council Oesophageal Cancer Working G. Surgical resection with or without preoperative chemotherapy in oesophageal cancer: a randomised controlled trial. Lancet 2002; 359:1727-33.

7. Sobin L, Wittekind C. TNM Classification of malignant tumours New-York. Wiley-Liss 2002

8. Greene FL, Page DL, Fleming ID, Fritz AG, Balch CM. AJCC Cancer Staging Manual. 6th ed. Springer-Verlag: New York 2002.

9. Lagarde SM, ten Kate FJ, Reitsma JB, Busch OR, van Lanschot JJ. Prognostic factors in adenocarcinoma of the esophagus or gastroesophageal junction. J Clin Oncol 2006; 24:4347-55.

10. de Manzoni G, Pedrazzani C, Verlato G, et al. Comparison of old and new TNM systems for nodal staging in adenocarcinoma of the gastro-oesophageal junction. Br J Surg 2004; 91:296-303.

11. Tanaka E, Hashimoto $Y$, Ito $T$, et al. The clinical significance of Aurora-A/STK15/BTAK expression in human esophageal squamous cell carcinoma. Clin Cancer Res 2005; 11:1827-34.

12. Polkowski W, van Sandick JW, Offerhaus GJ, et al. Prognostic value of Lauren classification and c-erbB-2 oncogene overexpression in adenocarcinoma of the esophagus and gastroesophageal junction. Ann Surg Oncol 1999; 6:290-7.

13. Walch AK, Zitzelsberger HF, Bink K, et al. Molecular genetic changes in metastatic primary Barrett's adenocarcinoma and related lymph node metastases: comparison with nonmetastatic Barrett's adenocarcinoma. Mod Pathol 2000; 13:814-24.
14. Saito H, Tsujitani S, Oka S, Ikeguchi M, Maeta M, Kaibara N. The expression of murine double minute 2 is a favorable prognostic marker in esophageal squamous cell carcinoma without p53 protein accumulation. Ann Surg Oncol 2002; 9:450-6.

15. Krishnadath KK, Tilanus HW, van Blankenstein M, et al. Reduced expression of the cadherin-catenin complex in oesophageal adenocarcinoma correlates with poor prognosis. J Pathol 1997; 182:331-8.

16. Sturm I, Petrowsky H, Volz R, et al. Analysis of p53/BAX/p16 (ink4a/CDKN2) in esophageal squamous cell carcinoma: high BAX and p16(ink4a/CDKN2) identifies patients with good prognosis. J Clin Oncol 2001; 19:2272-81.

17. Masuda N, Kato H, Nakajima T, et al. Synergistic decline in expressions of $\mathrm{p} 73$ and $\mathrm{p} 21$ with invasion in esophageal cancers. Cancer Sci 2003; 94:612-7.

18. Ewart-Toland A, Briassouli P, de Koning JP, et al. Identification of Stk6/STK15 as a candidate low-penetrance tumor-susceptibility gene in mouse and human. Nat Genet 2003; 34:403-12.

19. Fleishman SJ, Schlessinger J, Ben-Tal N. A putative molecularactivation switch in the transmembrane domain of erbB2. Proc Natl Acad Sci U S A 2002; 99:15937-40.

20. Bond GL, $\mathrm{Hu} \mathrm{W}$, Bond $\mathrm{EE}$, et al. A single nucleotide polymorphism in the MDM2 promoter attenuates the p53 tumor suppressor pathway and accelerates tumor formation in humans. Cell 2004; 119:591-602.

21. Shin Y, Kim IJ, Kang HC, et al. The E-cadherin $-347 \mathrm{G}->\mathrm{GA}$ promoter polymorphism and its effect on transcriptional regulation. Carcinogenesis 2004; 25:895-9.

22. Conne B, Stutz A, Vassalli JD. The 3' untranslated region of messenger RNA: A molecular 'hotspot' for pathology? Nat Med 2000; 6:637-41.

23. Kaghad M, Bonnet H, Yang A, et al. Monoallelically expressed gene related to $\mathrm{p} 53$ at $1 \mathrm{p} 36$, a region frequently deleted in neuroblastoma and other human cancers. Cell 1997; 90:809-19.

24. Heist RS, Zhou W, Chirieac LR, et al. MDM2 polymorphism, survival, and histology in early-stage non-small-cell lung cancer. J Clin Oncol 2007; 25:2243-7.

25. Boersma BJ, Howe TM, Goodman JE, et al. Association of breast cancer outcome with status of p53 and MDM2 SNP309. J Natl Cancer Inst 2006; 98:911-9.

26. Cescon DW, Bradbury PA, Asomaning K, et al. p53 Arg72Pro and MDM2 T309G polymorphisms, histology, and esophageal cancer prognosis. Clin Cancer Res 2009; 15:3103-9.

27. Nakamura A, Shimazaki T, Kaneko K, et al. Characterization of DNA polymorphisms in the E-cadherin gene $(\mathrm{CDH} 1)$ promoter region. Mutat Res 2002; 502:19-24. 